\title{
Borrelia Burgdorferi Infection
}

National Cancer Institute

\section{Source}

National Cancer Institute. Borrelia Burgdorferi Infection. NCI Thesaurus. Code C45161.

An infectious disease caused by the spirochete Borrelia burg dorferi. Early manifestations

of infection may include fever, headache, fatigue, depression, and a characteristic skin rash called erythema migrans. Left untreated, late manifestations involving the joints, heart, and nervous system can occur. 